\title{
Engineering Laboratory Accessible via the Internet
}

Session 1526

\author{
Victor Skormin, Vladimir Nikulin \\ Binghamton University, State University of New York
}

\begin{abstract}
This paper presents a system facilitating remote multiple-user access to advanced laboratory instrumentation via the Internet. The technique itself, and its potential for the enhancement of engineering laboratory courses and, consequently, engineering programs nationwide and internationally is discussed.
\end{abstract}

\section{Introduction}

The on-going revolution in information technology results in noticeable advancements in university education. These advancements come at the right time: the amounts of knowledge expected at the baccalaureate and master's levels show drastic increase. The system of engineering education is especially vulnerable to the effects of Internet, global communication systems, computers, etc. However, there is one area in engineering education that is still dominated by classical teaching/learning methodology: the laboratory. This could be easily explained: the purpose of an engineering laboratory course is to teach future engineers to interact with the "real hardware" in all its imperfection. Any attempt to replace the "real hardware" in a student laboratory with the most elaborate simulation software can result in the loss of realism and prevents students from gaining important practical skills and experiences. Unfortunately, modern engineering laboratory equipment is highly expensive. In addition, the maintenance and repair costs, along with the floor space requirements, often exceed the resources of many universities. This provides the only argument in support of educational laboratory utilizing virtual reality techniques: virtual reality is better than no reality at all.

The technology presented herein is not a virtual reality laboratory. It utilizes advanced spacequalified laser positioning hardware equipped with computer interfaces facilitating remote operation and status display of its components. All aspects of operation of this hardware are controlled by a designated computer through a number of actuators and extensive monitoring/data acquisition. This system has been upgraded to achieve global accessibility of the "real hardware" via the Internet thus allowing remote users worldwide to perform any experiments in real-time and collect feedback information representing properties of the actual devices. A special effort is being made to provide the user with a view of the laboratory setup from several positions. This would bring to a student laboratory the most valuable aspects of the "real hardware"-based experiments. It is believed that the choice of laboratory, laser steering and position control systems for space communication, will make it attractive to many engineering programs. Successful implementation of this technology would upgrade any 
engineering laboratory to the level of leading engineering schools and result in significant improvement of the quality of engineering education and reduce its variability between universities. The presented approach has many potential applications, including training of personnel to operate complex machinery in hazardous environments.

Being prompted by revolutionary changes in information technology, this project will result in the further integration of this technology in education. Implementation of the Internet-accessible laboratory courses and training programs will not only give students access to the most advanced hardware, but provide them with an additional opportunity to utilize Internet, computer graphics, and digital imaging thus preparing them for the challenges of their profession.

\section{Laboratory Courses in Engineering Education}

Laboratory courses constitute a very important component of engineering education. Authorities in the area of methodology of engineering education emphasize the role of student laboratory in achieving such educational goals as experimental skills, sense of real world, taste of discovery, understanding equipment, motivation, appreciation of the power of team work, networking skills, communication skills, and the importance of independent learning [1], [2], [3], [4]. Indeed, only in a laboratory course can students design, implement and later assess a plan of an experiment leading to the solution of the formulated problem, that includes development of an experimental setup, choosing a rational sequence of stimuli, recording, analyzing and interpreting data. Student laboratory provides a demonstration of the power of "poor connections", "second order effects", "hidden dynamics", measurement noise, effects of overheating, cross-talk between wires, etc. Indeed, some of our colleagues are convinced that the imperfection of hardware results in the most profound educational effect on future engineers. A well-designed laboratory experiment presents students with uncertainty, non-trivial outcome, and an opportunity of discovering new, not mentioned in a textbook, properties and phenomena. It is said that complex engineering systems and instruments, being sensitive to many hidden environmental effects, "live their own lives", and the ability to understand equipment is a long, sometimes life-long, process that starts in a student laboratory. Only the motivation of a specialist can overcome Murphy's law, measurement noise, unexpected behavior of hardware, etc. and obtain the needed results from the available equipment under existing conditions. Unlike theoretical problems reflecting only the simplified quantitative side of the "real thing", a laboratory assignment deals directly with "real things" in all their complexity and imperfection thus providing the obvious need for team work, illustrating the importance of forming the right team, distribution and coordination of tasks and assignments, interaction and cooperation between team members, negotiation and exchange, that ultimately results in the development of networking and communication skills highly appreciated by employers. Finally, student laboratory presents an opportunity to observe how abstract theories and laws manifest (or do not manifest) themselves in operation of particular devices that reinforces theoretical background and provides the motivation for independent learning.

While laboratory courses are crucial for the success of future engineers, it is known that poorly equipped and trivial laboratory experiments "send a wrong message to students" and can do more harm than good outside the protective campus walls. Unfortunately, modern laboratory 
equipment consistent with the state-of-the-art engineering and research facilities is highly expensive. It is also very "touchy", requires intricate adjustment, and should be operated by well-trained personnel. In the hazardous environment of a student laboratory, this equipment has a very short life span and must be often serviced, repaired and replaced. Its maintenance, repair and replacement costs, along with the floor space requirements, often exceed the resources of many universities. While the consistent theoretical level of the same courses taught at different universities could be assured by the use of the same textbooks, course requirements and examination procedures, available laboratory equipment is one of the major factors responsible for the variability of quality of engineering education between universities.

Engineering educators have reached a consensus that computer simulation, even virtual reality, does not provide a substitute for traditional "hardware-based" laboratory courses. Indeed, by definition, a laboratory experiment is intended to demonstrate the complexity and some degree of unpredictability of the real physical phenomena, not just the "first order effects" implemented in simulation software. The conclusion is straightforward: high quality of engineering education cannot be achieved without providing students access to an appropriately equipped engineering laboratory.

\section{The System Configuration}

Any laboratory experiment is intended to provide the students with an opportunity to visualize the laboratory hardware, to learn about its components, their functions and principles of operation, to establish the goal of the experiment, to design the experiment, to apply stimuli to the laboratory setup, to observe the hardware in action, to record data featuring the stimuli and the response of hardware, and, finally, to process the recorded data. Therefore, the system that is being developed must address all these functions utilizing the power of the Internet and computer technology to their full potential.

The visualization is achieved by compiling a library of digital photographs of the laboratory setup taken from different positions and angles of view and establishing a graphic interface that allows the remote user to "rotate" the setup in 3D. In order to learn about system components, the user is expected to point a curser at the appropriate area within the digital photograph that will result in a special window providing a textbook-quality description of the relevant system component(s). Although we intend to provide the user with a number of suggested laboratory experiments, we would encourage our remote users to pursue their own research goals and design their own experiments. Indeed, the role of a "lender of hardware" is even more consistent with the proposed technology than just the one of an educator. This perception drastically widens the functions of an Internet-accessible laboratory that could be appreciated by practicing engineers and researchers worldwide.

Any engineering experiment implies application of some strategically defined stimuli and recording the system responses. Our system has the facilities for the generation of typical input signals, such as step, ramp, Gaussian noise, sinusoidal and square waves, and offers the choice of their parameters. In addition, a user can apply his/her own input signals recorded in a data file. It is important though that specially designed software routines would automatically limit 
magnitudes and frequencies of the input signals protecting the laboratory hardware from possible damage. Numerically defined inputs are eventually represented by data files featuring magnitudes and time steps of the corresponding "stair case-type" voltage signals generated by the auxiliary electronics and applied to the actuators of the laboratory setup.

The ability to observe the response of the laboratory setup to the applied stimuli provides a very important learning experience. While this ability not necessarily exists when dealing with real hardware, due to very short transient response time or very small physical changes that take place, we assure this ability by the use of animated schematics that operates on a specially chosen tame scale thus providing some reward to a remote user for not being present in the laboratory (similarly, the ability to see a sports event in all detail is a TV viewer's reward for not being present at the stadium). The response of laboratory hardware, converted by a number of sensors and computer interfaces in voltage signals and recorded in a data file becomes immediately available to the user who has the ability to utilize data processing and plotting facilities or/and download this data file for further processing.

The system development efforts include: website design, JavaScript programming, programming in $\mathrm{C}^{++}$using CGI standard, working with Data Acquisition boards, building dynamic link libraries, extending commercial applications. The developers are guided by the following considerations,

- the system hardware and software must be generic and suitable for the application to various laboratory setups

- the system could be easily upgraded by replacing/adding new modules

- the software-hardware interface utilizes commercially-available software such as VISSIM [5], and potentially, Lab-VIEW [6]

The following schematic represents the overall configuration of a laboratory setup accessible via the Internet.

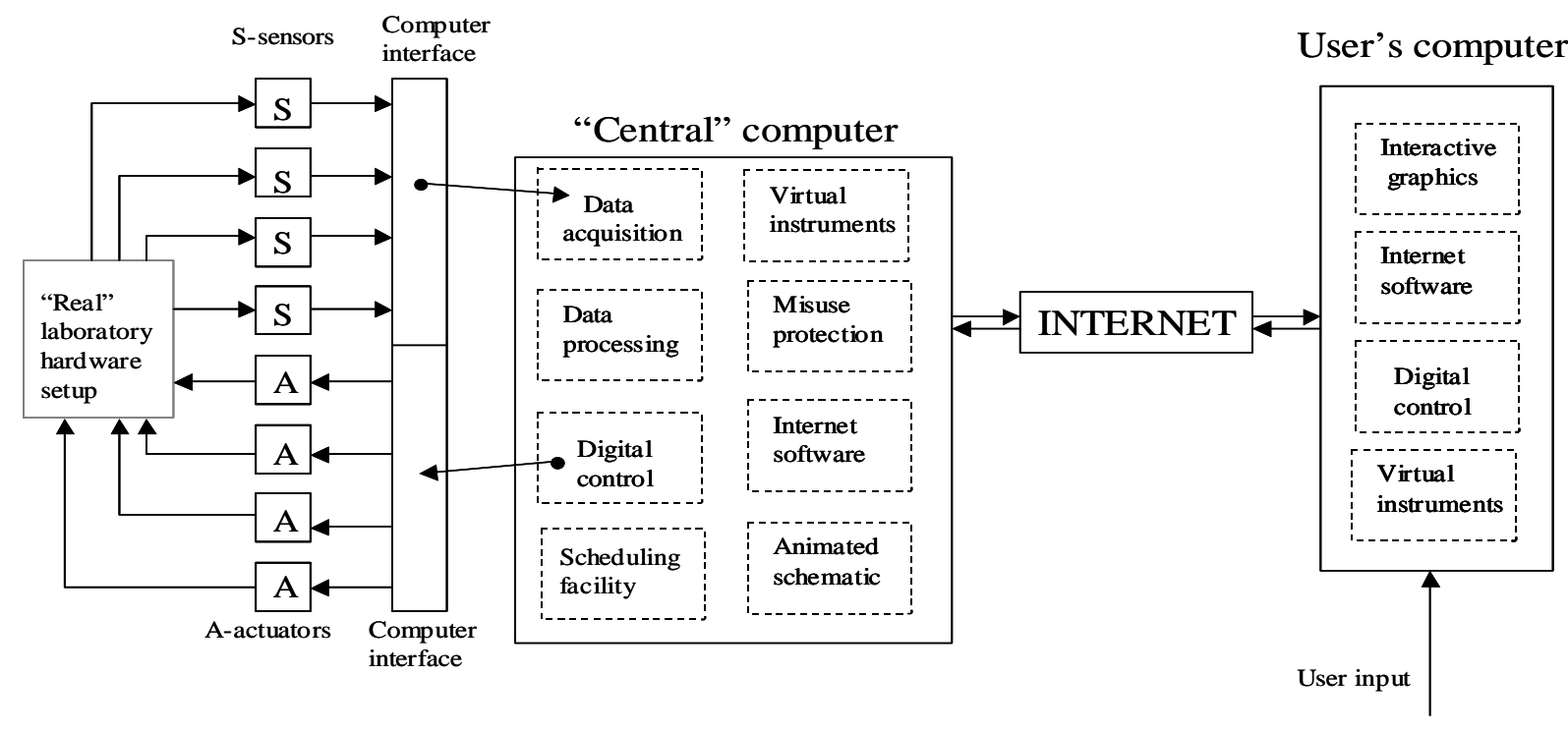

Proceedings of the 2001 American Society for Engineering Education Annual Conference \& Exposition Copyright @ 2001, American Society for Engineering Education 
It could be seen that a "real" hardware setup is interfaced with a "central" computer that, operating through a system of actuators (A) and sensors (S), performs control and monitoring tasks. The following software modules are deployed on the central computer:

- Monitoring/data acquisition system recording real-time sensory data representing the actual status of the hardware (accelerations and positions of moving mechanical parts, voltage and current signals, etc.)

- Digital control system responsible for the generation of control efforts, primarily in the form of voltage signals, defined by the user, or internally on the basis of some control laws. In addition, the control system is capable of closing (or opening) the control loop and implementing various control laws

- Animated schematic is a software module implementing computer graphics that displays the time-varying status of the laboratory hardware featuring positions of all moving mechanical parts and illustrating propagation of laser beams, mechanical vibrations, and electrical signals. In order to achieve a better visualization of the experiment, animated schematics will be supplemented by real-time digital imaging (note that in many instances digital imaging will be less informative than animated schematics)

- Data processing module includes software routines performing spectral analysis, correlation analysis, filtering, averaging, and integration of signals.

- Virtual instruments module will emulate common laboratory instruments, such as multimeters, oscilloscopes, recorders, plotters, etc. that, using graphic interface, could be "taken off the shelf" and "installed" to characterize particular signals of interest

- Misuse protection module will be responsible for protecting the hardware from potential damage by the user applying excessively high magnitude signals or inappropriate frequency signals that could cause resonance; this module will interrupt the experiment in the case of equipment overheating, inadequate power supply, etc.

- Scheduling facility will perform the necessary functions that will facilitate the use of the laboratory setup by multiple users (one user at a time) according to some system of priorities, requested date, time and duration of the experiment

- Internet software will be responsible for the interfacing with the Internet and the consequent data communication

On the user's side, the software configuration is as follows:

- Interactive graphics will utilize computer graphics and real-time data transmitted over the Internet to illustrate the status of the laboratory setup including varying positions of moving mechanical parts, laser beams, propagation of relevant signals, etc. In order to minimize the amount of data to be transmitted, the animated schematics software must be downloaded to the user's computer, and then be driven via the Internet. In order to enhance the visualization of the experiment, the user will have the opportunity to utilize the real-time digital imaging facility

- Digital control facility will allow a remote user to configure the system (opened-loop or closed-loop, choice of particular components). In the opened-loop case, the user will have the opportunity to specify types and characteristics of the control efforts that will be applied directly to the hardware. In the closed-loop case, the user will be capable of choosing between several control laws (PID, state-variable), applying the desired parameters of the control law, and then applying the desired reference signal (step, sinusoid, etc.) 
- Virtual instruments software, downloaded to the user's computer, will facilitate handling and display of the real-time data that could be subjected to further processing

- Internet software will be responsible for interfacing with the Internet, scheduling access to the laboratory setup, and consequent data communication.

Not shown in the figure, is the software facility that would provide the user with a hardware manual, and the description of the suggested experiments.

\section{The Working Prototype}

The working prototype of a laboratory setup accessible via the Internet, providing the proof-ofconcept, is being developed in the Laser Communication Laboratory of the Watson School of Engineering at Binghamton University under NSF funding. Under the terms of the Project it will be subjected to testing and evaluation at several universities, SUNY at Stony Brook, Purdue University Indianapolis, and Ben-Gurion University of the Negev in Israel (the authors would gladly expand this list). A computer-driven space-qualified piezo-electric optical mirror [7], [8] intended for laser communications that has been chosen for the prototype development. This choice is based upon the realization that

- laser communication is an emerging technology that is of interest to many electrical engineering programs nationwide and internationally,

- the mirror is a spatially-distributed structure that exhibits noticeable inertia, several resonant modes, cross-coupling between azimuth and elevation channels, and nonlinear behavior, its experimental characterization and mathematical modeling constitutes a very interesting task for both electrical and mechanical engineers,

- operation of this beam steering mirror in a realistic environment results in a complex mechanical phenomena that could be of interest to mechanical engineers specializing in active and conventional vibration control,

- performance of this mirror (frequency- and time-domain characteristics, decoupling of cross-coupled channels) can be greatly enhanced by designing a successful feedback controller that presents a challenging task for electrical engineers specializing in controls.

The following figure demonstrates one of the screens intended for a user conducting experiments on the piezo-electric optical mirror via the Internet. The computer, not shown in the figure, generates voltage signals that after amplification are applied to piezo-electric actuators resulting in the appropriate azimuth and elevation components of the mirror deflection. The mirror deflection results in the repositioning of the laser beam on the array detector that provides the accurate information on the actual beam position. The computer-based monitoring system records the voltage signals driving the mirror, azimuth and elevation components of the mirror deflection, and/or the azimuth and elevation components of the beam position. In addition, the computer allows for the introduction of a mirror (and/or laser beam) position feedback and implementation of various control laws. 


\section{Experiment Parameters:}

Channel 1:

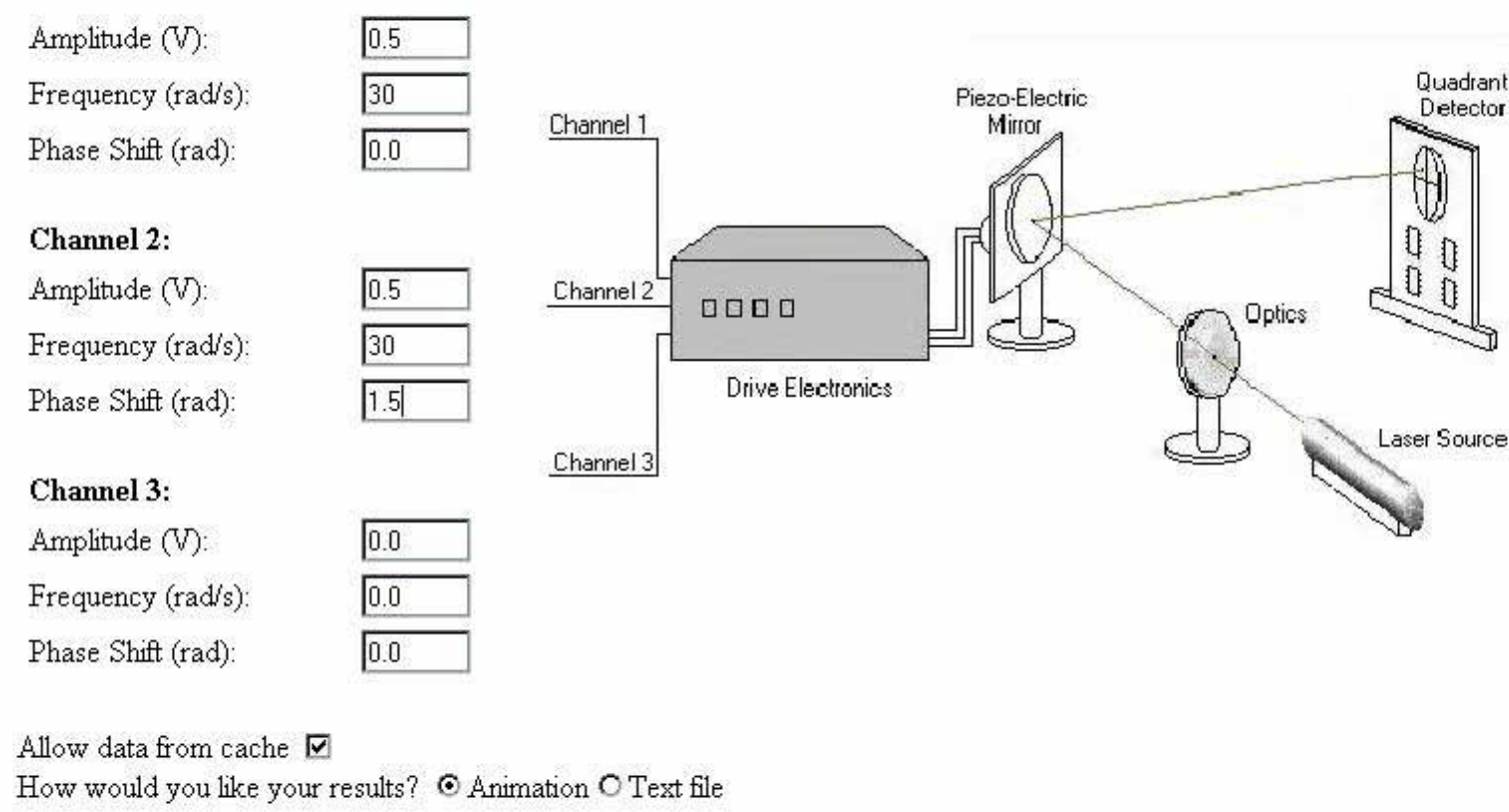

Start

The readers are invited to visit the authors' website http://www.ee.binghamton.edu/lasercom/ and observe the developed system in more detail (please note that, first, the system is only at its developmental stage and, second, an experiment could be conducted only when the laboratory setup is turned on).

\section{Recommended Experiments}

Characterization of high-performance instrumentation is a task typical for a practicing electrical or mechanical engineer. The remotely-operated laboratory setup is ideal for obtaining data that being processed would result in a frequency response of the direct and cross-channels of the optical mirror with the emphases on such important characteristics as bandwidth, resonant frequencies, and cross-coupling between channels. Similarly, time response of the direct and cross-channels could be recorded, plotted and analyzed for the settling time, natural frequencies, overshoot, damping and cross-coupling phenomena. The students could be challenged by the choice of input signals, duration of the experiment, choice of signal processing procedures. Evaluation of the nonlinear effects in the mirror response and establishing a linear range of operation could be recommended as a more advanced assignment. 
Mathematical modeling of the optical mirror could be the next task. Recoded experimental data would provide information for establishing a transfer matrix of the optical mirror resulting in a complete mathematical description. Linear and nonlinear least squares procedures could be successfully applied for parameter estimation; combined with the use of F-criterion and coefficient of determination, they will result in a simple and statistically valid mathematical model of the mirror. This mathematical model, implemented in simulation software, could be subjected to the same stimuli as the "real mirror". Comparative analysis of the actual and simulated responses would provide important insight for the understanding of underlying physical phenomena and further validation and fine-tuning of the model.

Finally, the remote users will utilize their ability to "close the loop" on the optical mirror and investigate the power of feedback control. Commonly, control system experiments include the analysis and synthesis tasks. The "analysis" experiment would imply introduction of some predefined feedback controllers and observing their effect on system frequency- and time-domain response. The "synthesis" experiment would require students to design a feedback controller to achieve the required system performance. It should be understood that the Internet cannot be a part of a control loop that must operate in "real time", and the user must be given access to control modules implemented in the "central" computer.

The appropriate lab manuals featuring the described experiments are being developed and will be available to the users of the Internet-accessible laboratory in the nearest future. At the same time, the authors want to re-iterate that the developed technology is intended as a means of providing access to advanced instrumentation worldwide, and its applications are not limited to engineering education.

\section{Acknowledgement}

The authors are grateful to Ivan Shaporov of Binghamton University for his intricate computer application efforts, to Dr. Timothy E. Busch of the Air Force Research Laboratory for his expert advice in the area of instrumentation, to Mounir Bouzoubaa of Binghamton University for help, advice and friendly discussions, and of course to the National Science Foundation for funding this research.

Bibliography

1. Wankat, P. C., Oreovicz, F. S., 'Teaching Engineering, ”McGraw-Hill, Inc., 1993

2. Eastlake, C. N., "Tell me, I'll forget; show me, I'll remember; involve me, I'll understand (The tangible benefit of labs in the undergraduate curriculum)," Proceedings ASEE Annual Conference, ASEE, Washington, DC, $420,1986$.

3. Jumper, E. J., "Recollections and observations on the value of laboratories in the undergraduate engineering curriculum," Proceedings ASEE Annual Conference, ASEE, Washington, DC, 423, 1986.

4. Radovich, J. M., "What is needed for a good laboratory program?", Engineering Education, 749, April 1983.

5. VisSim Software, Visual Solutions, Inc., Westford, MA 01886 
6. LabVIEW Graphical Programming for Instrumentation, NATIONAL INSTRUMENTS, Austin, TX, Copyright 1992

7. R. Dumas and B. Laurent, "System Testbed for Demonstration of the Optical Space Communications Feasibility", SPIE Vol. 1218, Free-Space Laser Communication Technologies II, 1990.

8. "An Experimental Spatial Acquisition and Tracking System for Optical Intersatellite Crosslinks", Technical Report prepared for the Department of the Air Force under contract F19628-90-C-0002, December 1991.

9. Skormin, V.A., Tascillo, M.A., Herman, C.R., and Nicholson, D.J., "Mathematical Modeling and Simulation Analysis of a Pointing, Acquisition and Tracking System for Laser-Based Intersatellite communication", Optical Engineering, November 93.

10. Skormin, V.A., Busch, T.E., and Tascillo, M.A., "An Adaptive Jitter Rejection Technique Applicable to Airborne Laser Communication System", Optical Engineering, 1995.

11. Skormin, V.A., Busch, T.E., Givens M.A., "Model Reference Control of a Fast Steering Mirror of a Pointing, Acquisition and Tracking System for Laser Communication", Proceedings of the National Aerospace Electronics Conference NAECON'95, Dayton OH,1995.

\section{VICTOR A. SKORMIN}

Victor A. Skormin is a Professor of Electrical Engineering at the Watson School of Engineering, Binghamton University (SUNY). His area of expertise includes control theory, system optimization, mathematical modeling, computer simulation, and laser communication. His research efforts includes development of components of laserbased space communication systems, control, optimization and diagnostic system for power plants, diagnostic systems for avionics, adaptive motion control systems. He served as a consultant to major US companies and the Air Force. He is actively involved in instructional TV and technical lecturing. Dr. Skormin is an author of many technical publications. His current research is funded by NSF, NASA, and Air Force. Dr. Skormin is a recipient of the IEEE Region I Award "For Leadership in Establishing University-Industry Links in Research and Education", the University Award for Graduate Teaching from Binghamton University, and the SUNY Chancellor's Award for Excellence in Teaching. He is a Senior Member of IEEE and served as the Editor for Space Systems of the IEEE Transactions on Aerospace and Electronic Systems. During 1999-2000 Dr. Skormin has been appointed as National Research Council's Senior Researcher with the Air Force. In 1999 Dr. Skormin was awarded the title of Honorary Professor of the Kazakh National Technical University, Almaty, Kazakhstan.

\section{VLADIMIR V. NIKULIN}

Vladimir V. Nikulin is a Research Scientist at the Laser Research Laboratory at the Watson School of Engineering, Binghamton University and a part-time Ph.D. student specializing in various aspects of advanced control engineering applied to pointing-acquisition-tracking components of laser communication. He is a recipient of a Binghamton University Graduate Student Excellence Award, and a Member of IEEE. 